\title{
ON THE SQUARES OF ORIENTED MANIFOLDS
}

\author{
R. E. STONG ${ }^{1}$
}

1. Introduction. The object of this paper is to give another proof of the Milnor conjecture [2]:

THEOREM 1. The square of an oriented manifold is unoriented cobordant to a Spin manifold.

This result was proved by Anderson [1]. The proof given here is patterned directly on the method used by Wall [4] in the determination of the oriented cobordism ring. This method provides additional information, and the principal result will be:

Theorem 2. Let $S^{\prime}$ denote the subset of $H^{*}\left(B O, Z_{2}\right)$ consisting of all classes $w_{2 i+1}$. Let $S$ be either $S^{\prime} \cup\left\{w_{2}\right\}$ or $S^{\prime} \cup\left\{w_{2}^{2}\right\}$. Let $M$ be a manifold such that every Stiefel-Whitney number of $M$ divisible by a class of $S$ is zero. Then:

(a) For $S=S^{\prime} \cup\left\{w_{2}^{2}\right\}, M$ is unoriented cobordant to a complex manifold $M^{\prime}$ with $c_{1}^{2}$ zero.

(b) For $S=S^{\prime} \cup\left\{w_{2}\right\}, M$ is unoriented cobordant to the sum of an $\mathrm{SU}$ manifold and a polynomial in the quaternionic projective spaces $\mathrm{QP}(2 n)$.

Theorem 1 is a direct consequence of the case $S=S^{\prime} \cup\left\{w_{2}\right\}$, and one has the improved result:

COROLlary. If $M$ is an oriented manifold with dimension not divisible by 4 , then $M \times M$ is unoriented cobordant to an SU manifold.

2. Proofs of the results. First consider the case $S=S^{\prime} \cup\left\{w_{2}^{2}\right\}$ and suppose $M$ has all Stiefel-Whitney numbers divisible by elements of $S$ zero.

By Milnor [2], $M$ is cobordant to a complex manifold $N$, since all numbers of $M$ divisible by elements of $S^{\prime}$ are zero. Then let $M^{\prime} \subset N$ $\times \mathrm{CP}(1)$ be a submanifold dual to $c_{1}(N)+\alpha, \alpha \in H^{2}(\mathrm{CP}(1), Z)$ being the usual generator. The total Chern class of $M^{\prime}$ is the restriction to $M^{\prime}$ of

$$
\frac{c\left(I^{\top}\right) \cdot(1+\alpha)^{2}}{1+\alpha+c_{1}(N)}=1+\alpha+\left(c_{2}(N)+\alpha c_{1}(N)\right)+\cdots,
$$

Received by the editors August 8, 1965.

1 The author is indebted to the National Science Foundation for financial support during this work. 
so $c_{1}^{2}\left(M^{\prime}\right)$ is the restriction to $M^{\prime}$ of $\alpha^{2}$, hence is zero. Reducing mod 2,

$$
w\left(M^{\prime}\right)=\frac{w(N)}{1+\alpha+w_{2}(N)},
$$

and $M^{\prime}$ is dual to $\alpha+w_{2}(N)$. Then for any $\omega=\left(i_{1}, \cdots, i_{r}\right), \sum i_{s}$ $=\operatorname{dim} M, w_{\omega}=w_{i_{1}} \cdots w_{i_{r}}$, one has

$$
w_{\omega}\left(M^{\prime}\right)=w_{\omega}(N)+\left(\alpha+w_{2}(N)\right) v_{\omega},
$$

where $v_{\omega}$ is a polynomial in $\alpha$ and the $w_{i}(N)$. Multiplying by $\left(\alpha+w_{2}(N)\right)$ and evaluating on $N \times \mathrm{CP}(1)$ gives

$$
\begin{aligned}
w_{\omega}\left[M^{\prime}\right] & =\left\{w_{\omega}(N) \cdot\left(\alpha+w_{2}(N)\right)+\left(\alpha+w_{2}(N)\right)^{2} v_{\omega}\right\}[N \times \mathrm{CP}(1)], \\
& =\left\{w_{\omega}(N) \cdot \alpha+w_{2}(N)^{2} v_{\omega}\right\}[N \times \mathrm{CP}(1)],
\end{aligned}
$$

and the second term is zero since every number of $N$ divisible by $w_{2}^{2}$ is zero. Then $w_{\omega}\left[M^{\prime}\right]=w_{\omega}[N]$ for all $\omega$, and by Thom [3], $M^{\prime}$ and $N$ are cobordant since they have the same Stiefel-Whitney numbers. This proves part (a) of the theorem.

Note. Using the same process with $\mathrm{RP}(1)$ and its class $\alpha$ gives a direct proof of Wall's result [4] that if $M$ has all numbers divisible by $w_{1}^{2}$ zero, then $M$ is cobordant to $M^{\prime}$ with $w_{1}^{2}=0$.

Let $W_{*}$ denote Wall's ring of cobordism classes for which numbers divisible by $w_{1}^{2}$ are zero. Since

$w_{i_{1}} \cdots w_{i_{r}}[N \times N]= \begin{cases}0 & \text { if any } i_{s} \text { is odd, } \\ w_{j_{1}} \cdots w_{j_{r}}[N] & \text { if } i_{s}=2 j_{s} \text { for all } s,\end{cases}$

one has $W_{*}^{2}=\left\{\alpha^{2} \mid \alpha \in W_{*}\right\}$ is the set of cobordism classes with numbers divisible by elements of $S^{\prime} \cup\left\{w_{2}^{2}\right\}$ zero.

Let $\partial_{1}: W_{*} \rightarrow W_{*}$ be the derivation defined by sending the cobordism class of $M$ to the class of a submanifold dual to $w_{1}$. If $\partial_{2}: W_{*}^{2} \rightarrow W_{*}^{2}$ : $\alpha^{2} \rightarrow\left(\partial_{1} \alpha\right)^{2}$, then $\partial_{2}$ is a derivation and sends the cobordism class of $M$ to the class of a manifold $N$ such that $w_{\omega}[N]=w_{2} \cdot w_{\omega}[M]$ for all $\omega$ (by the formula for $w_{\omega}[A \times A]$ ).

Now if $\alpha^{2} \in W_{*}^{2}$, let $M$ be a complex manifold with $c_{1}^{2}=0$ belonging to $\alpha^{2}$. Let $N$ be a submanifold dual to $c_{1}$ in $M$. Then $w_{\omega}[N]=w_{2}$ - $w_{\omega}[M]$ for all $\omega$, so $N$ belongs to $\left(\partial_{1} \alpha\right)^{2}=\partial_{2}\left(\alpha^{2}\right)$, and $N$ is an SU manifold. Thus Im $\partial_{2}$ consists entirely of cobordism classes of SU manifolds.

By Wall [4], $\operatorname{ker} \partial_{1} / \operatorname{Im} \partial_{1}$ is the $Z_{2}$ polynomial algebra on the images of the cobordism classes of the complex projective spaces $\mathrm{CP}(2 n)$, so ker $\partial_{2} / \operatorname{Im} \partial_{2}$ is the $Z_{2}$ polynomial algebra on the images 
of the cobordism classes of the $\mathrm{CP}(2 n)^{2}$ (which is the same as that of $\mathrm{QP}(2 n))$.

Now let $M$ be any manifold all of whose Stiefel-Whitney numbers divisible by elements of $S^{\prime} \cup\left\{w_{2}\right\}$ are zero. Then the cobordism class $\alpha$ of $M$ belongs to ker $\partial_{2}$. Thus there is a polynomial $P$ in the $Q P(2 n)$ with cobordism class $\gamma$ for which $\alpha-\gamma \in \operatorname{Im} \partial_{2}$. Hence there is an SU manifold $N$ such that $M$ is cobordant to $N+P$, proving part (b) of Theorem 2.

Note. ker $\partial_{2}=\left\{\alpha^{2} \mid \alpha \in\right.$ ker $\left.\partial_{1}\right\}$ is precisely the cobordism classes of squares of oriented manifolds.

\section{REFERENCES}

1. P. G. Anderson, Cobordism classes of squares of orientable manifolds, Bull. Amer. Math. Soc. 70 (1964), 818-819.

2. J. Milnor, On the Stiefel-Whitney numbers of complex manifolds and of spin manifolds, Topology 3 (1965), 223-230.

3. R. Thom, Quelques propriêtés globales des variêtés différentiables, Comment. Math. Helv. 28 (1954), 17-86.

4. C. T. C. Wall, Determination of the cobordism ring, Ann. of Math. (2) 72 (1960), $292-311$.

Mathematical Institute, Oxford University 